\title{
Quantitative Structure-Activity Relationship Study of Curcumin Analogues as Anti-proliferative Agents of Human Prostate Cancer Cell Line [PC-3]
}

\author{
Andrea Marie M. Reyes and Junie B. Billones* \\ Department of Physical Sciences and Mathematics \\ College of Arts and Sciences \\ University of the Philippines Manila \\ Padre Faura, Ermita, Manila 1000 Philippines
}

\begin{abstract}
A quantitative structure-activity relationship (QSAR) study has been performed on curcumin analogues in order to establish the effect of variation of structural, electronic and topological properties on the anti-proliferative activity against prostate cancer cell line PC-3. The structure-based properties were calculated by the use of Hyperchem ${ }^{\circledR}$ and Dragon ${ }^{\circledR}$ softwares and the multi-linear regression equations were generated by the use of SPSS ${ }^{\circledR}$ software. Six models of PC-3 activity have been developed according to the types of QSAR descriptors. All models were cross-validated using leave-one-out (LOO) method. The models indicate that a more potent curcumin derivative against PC-3 should have smaller surface area, greater volume, lower polarizability, and fewer oxygen and multiple bonds.
\end{abstract}

Keywords: curcumin; PC-3 prostate cancer; quantitative structure-activity relationship study

\section{INTRODUCTION}

Many metabolically active components that are derived from medicinal or dietary plants, known as phytochemicals, possess chemopreventive properties. Curcumin, the active compound of the spice turmeric, has been heavily studied during these past few decades (Anand et al., 2008; Srivastava et al., 2011). It is a relatively non-toxic polyphenol, that possesses anti-inflammatory, anti-oxidant, and anti-septic effects (Hergenhahn et al., 2002). It also exhibits anticancer activity in many human cancer cell lines (Chathoth et al., 2008). Curcumin was reported to be beneficial in all 3 stages of carcinogenesis, namely, initiation, promotion, and progression. It seems to be one of the most promising treatments of cancer (Aggarwal et al., 2003).

Curcumin has been shown to affect many cellular and molecular pathways. The mechanisms implicated are diverse and appear to involve a combination of cell signaling pathways at multiple levels (Sa et al., 2010). It can suppress cancer cell proliferation, induce apoptosis, inhibit angiogenesis, and suppress the expression of anti-apoptotic proteins while protecting immune system of the tumor bearer (Campbell and Collett, 2005). 
Moreover, curcumin analogues have been synthesized and tested against PC-3 prostrate cancer cell line (Fuchs et al., 2009).

A quantitative structure-activity relationship study on these analogues would allow the identification of molecular properties that contribute to curcumin's inhibitory activity against PC-3. The availability of models relating activity and molecular properties would facilitate the design and development of more potent curcumin derivatives against prostate cancer. Thus, this study aims to establish quantitative relationships between the molecular properties of 24 curcumin analogues and their prostate cancer growth inhibitory activities. Specifically, the molecular properties such as physical, functional group counts, topological, geometric, constitutional, and electrotopological properties of each curcumin analogue were calculated. Subsequently, model equations for inhibitory activity were developed using multilinear regression analysis and validated using leaveone-out (LOO) method (Gong, 1986).

\section{METHODOLOGY}

A data set of 24 curcumin analogues and their biological activity against the PC3 cancer cell lines were obtained from existing literature [8]. The experimental $\mathrm{IC}_{50}$ values of curcumin analogues used in this work were reportedly obtained under the same set of conditions. Figure 1 shows the structure of curcumin and Table 1 gives the list of curcumin analogues along with their respective $\mathrm{IC}_{50}$ values.

The two-dimensional structures of the analogues were drawn using the HyperChem software (Hypercube, Inc., 2011) running in

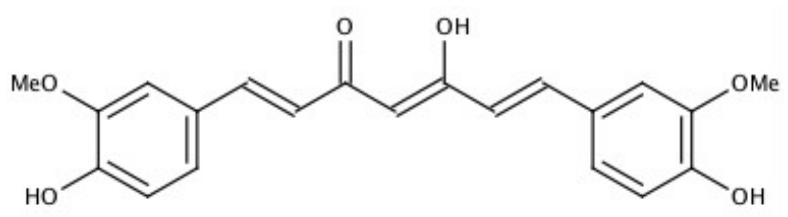

Figure 1. Chemical structure of curcumin
Windows 7 OS. Each structure was geometrically optimized at semi-empirical Austin Method 1 level. The resulting structures were used as input in subsequent property calculations in the Dragon (Tetko et al., 2005) and preADMET (Lee et al., 2004) programs. Linear models of PC-3 activities (the dependent variables) in terms of calculated molecular properties (independent variables or descriptors) were generated using SPSS $^{\circledR}$ (version 17) Statistics software.

The physicochemical parameters such as surface area, mass, $\log \mathrm{P}$, polarizability, refractivity, volume, hydration energy, dipole moment, highest occupied molecular orbital (HOMO), and lowest unoccupied molecular orbital (LUMO) energies were determined using Hyperchem. The other descriptors, hardness, softness, electrophilicity and absolute electronegativity were computed from HOMO and LUMO values according to the method proposed by Thanikaivelan et al. (Thanikaivelan et al., 2000). The Dragon program on the other hand was used to calculate over a thousand different functional groups, topological, charge, quantum, and constitutional descriptors for each molecule. Dragon ${ }^{\circledR}$ is a cheminformatics application for the calculation of thousands of molecular descriptors (e.g. constitutional and ring descriptors; topological, connectivity, and information indices; walk and path, and functional group counts, etc.). These descriptors can be used to evaluate molecular structure-activity or structure-property relationships, as well as for similarity analysis and high-throughput screening of molecule databases. PreADMET software was also used to calculate for geometrical and electrotopological charge indices descriptors.

The generated models were validated by leave one out cross validation procedure. This procedure involves leaving one molecule out and then a model is generated based on $n-1$ dataset. The model generated is used to calculate the predicted $\mathrm{IC}_{50}$ of the molecule that was left out. The same procedure is done for each analogue. The set of resultant predicted $\mathrm{IC}_{50}$ is then plotted against the 
Table 1. Curcumin Analogues and their Experimental $\mathrm{IC}_{50}(\mu \mathrm{M})$ and $\mathrm{pIC}_{50}$ values [8].<smiles>[Y]c1cc(/C=C/C(O)=C/C(=O)/C=C/c2cc(N)c([R])c(N)c2)cc(N)c1[R]</smiles>

1-9<smiles>COc1cc(/C=C/c2cc(/C=C/c3ccc(O)c(OC)c3)n([O-])n2)ccc1O</smiles>

12-13<smiles>COc1cc(OC)c(/C=C/C(=O)/C=C/c2c(OC)cc(OC)cc2OC)c(OC)c1</smiles>

23<smiles>COc1cc(/C=C/C(O)=C/C(=O)/C=C/c2ccc([N+](=O)[O-])c(O)c2)ccc1Br</smiles>

10-11

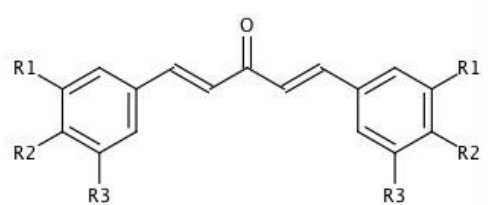

$14-22$<smiles>COc1cc(OC)c(OC)cc1/C=C/C(=O)/C=C/c1cc(OC)c(OC)cc1OC</smiles>

24

\begin{tabular}{|c|c|c|c|c|c|c|c|}
\hline Compound & $\mathbf{R}_{1}$ & $\mathbf{R}_{2}$ & $\mathbf{R}_{3}$ & $\mathbf{R}_{4}$ & $\mathbf{R}_{5}$ & $\begin{array}{c}\text { PC-3 IC } \text { I }_{50} \\
(\mathrm{mM})\end{array}$ & $\begin{array}{l}\text { PC-3 } \\
\text { pIC }_{50}\end{array}$ \\
\hline curcumin & $\mathrm{OCH}_{3}$ & $\mathrm{OH}$ & $\mathrm{H}$ & - & - & 19.8 & -1.297 \\
\hline 1 & $\mathrm{OCH}_{3}$ & $\mathrm{H}$ & $\mathrm{H}$ & - & - & 40 & -1.60 \\
\hline 2 & $\mathrm{H}$ & $\mathrm{OH}$ & $\mathrm{H}$ & - & - & 27.3 & -1.436 \\
\hline 3 & $\mathrm{OH}$ & $\mathrm{OCH}_{3}$ & $\mathrm{H}$ & - & - & 40 & -1.60 \\
\hline 4 & $\mathrm{OCH}_{3}$ & $\mathrm{OH}$ & $\mathrm{OCH}_{3}$ & - & - & 37.2 & -1.571 \\
\hline 5 & $\mathrm{OCH}_{3}$ & $\mathrm{OSO}_{2} \mathrm{NH}_{2}$ & $\mathrm{H}$ & - & - & 7.5 & -0.88 \\
\hline 6 & $\mathrm{OCH}_{3}$ & $\mathrm{OCH}_{3}$ & $\mathrm{H}$ & - & - & 5.9 & -0.77 \\
\hline 7 & $\mathrm{OAc}$ & $\mathrm{OAc}$ & $\mathrm{H}$ & - & - & 12.9 & -1.111 \\
\hline 8 & $\mathrm{OCH}_{3}$ & $\mathrm{OSO}_{2} \mathrm{NH}_{2}$ & $\mathrm{OCH}_{3}$ & - & - & 13.1 & -1.117 \\
\hline 9 & $\mathrm{OSO}_{2} \mathrm{NH}_{2}$ & $\mathrm{OCH}_{3}$ & $\mathrm{H}$ & - & - & 7.4 & -0.87 \\
\hline 10 & - & - & - & $\mathrm{OCH}_{3}$ & $\mathrm{OH}$ & 20.9 & -1.320 \\
\hline 11 & - & - & - & $\mathrm{OSO}_{2} \mathrm{NH}_{2}$ & $\mathrm{OH}$ & 193 & -2.286 \\
\hline 12 & - & - & - & $\mathrm{H}$ & 5.6 & -0.75 & 12 \\
\hline 13 & - & - & - & $\mathrm{CH}_{3}$ & 16.2 & -1.210 & 13 \\
\hline 14 & $\mathrm{OCH}_{3}$ & $\mathrm{OH}$ & $\mathrm{H}$ & - & 3.9 & -0.59 & 14 \\
\hline 15 & $\mathrm{OH}$ & $\mathrm{OCH}_{3}$ & $\mathrm{H}$ & - & 5.9 & -0.77 & 15 \\
\hline 16 & $\mathrm{H}$ & $\mathrm{OH}$ & $\mathrm{H}$ & - & 9.5 & -0.98 & 16 \\
\hline 17 & $\mathrm{OCH}_{3}$ & $\mathrm{OCH}_{3}$ & $\mathrm{H}$ & - & 2.9 & -0.46 & 17 \\
\hline 18 & $\mathrm{OCH}_{3}$ & $\mathrm{H}$ & $\mathrm{OCH}_{3}$ & - & 2.5 & -0.40 & 18 \\
\hline 19 & $\mathrm{OCH}_{3}$ & $\mathrm{OH}$ & $\mathrm{OCH}_{3}$ & - & 3.6 & -0.56 & 19 \\
\hline 20 & $\mathrm{OCH}_{3}$ & $\mathrm{OSO}_{2} \mathrm{NH}_{2}$ & $\mathrm{H}$ & - & 6.1 & -0.79 & 20 \\
\hline 21 & $\mathrm{H}$ & $\mathrm{OSO}_{2} \mathrm{NH}_{2}$ & $\mathrm{H}$ & - & 5.1 & -0.71 & 21 \\
\hline 22 & $\mathrm{OCH}_{3}$ & $\mathrm{OSO}_{2} \mathrm{NH}_{2}$ & $\mathrm{OCH}_{3}$ & - & 2.4 & -0.38 & 22 \\
\hline 23 & - & - & - & - & 2.1 & -0.32 & 23 \\
\hline 24 & - & - & - & - & 4.6 & -0.66 & 24 \\
\hline
\end{tabular}


experimental $\mathrm{IC}_{50}$. To test for collinearity, the descriptors were also analyzed using the bivariate correlation procedure.

\section{RESULTS AND DISCUSSION}

In this work, we aimed to generate models comprising of variables of same class or category because a model based on a particular class of descriptors can be easily applied in future structure-activity relationship studies. For example, it is easy to extend the results of a modeling study based on topological descriptors only because there are freely available programs that can calculate this particular class of descriptors. On the other hand, the application of a "general model", which may embody diverse type of descriptors, will require more and diverse computational resources.

Incidentally, the calculated molecular properties obtained by the use of Hyperchem, Dragon and preADMET softwares can be grouped into six classes, namely, physical, constitutional, functional group counts, topological, electrotopological state (E-state), and geometric descriptors. Hence, six sets of descriptors were obtained and consequently six model equations of anticancer activity were generated using SPSS statistics program.
At the outset, examination of the dataset prompted us to eliminate analogue $\mathbf{1 1}$ because its $\mathrm{IC}_{50}$ value $(193.00 \mathrm{mM})$ is significantly different from the rest of the analogues whose $\mathrm{IC}_{50}$ values range from 2 to $37 \mathrm{mM}$, otherwise it would constitute an outlier and affects the quality of the regression models. Specifically, the inclusion of analogue $\mathbf{1 1}$ in the data set gave an unreasonably high correlation coefficient. Its effect was like reducing the data set to two points, which is of course a straight line. Moreover, with only a score of samples at hand, we limited the number of independent variables in the model to only five (Tabachnick and Fidell, 2006). The final predictors that constituted the models were obtained by the use of the backward elimination protocol in SPSS.

Among the six models developed for PC-3 activity, the geometrical descriptors model gave the best statistics with non-crossvalidated squared correlation coefficient $\left(r^{2}\right)$ of 0.80 and cross-validated or predictive squared correlation coefficient $\left(q^{2}\right)$ of 0.75 . The dependence of PC-3 activity on geometrical descriptors is defined in Equation 1.

This equation shows that the inhibitory capacity of the curcumin analogues depends on the hydrophobic surface area (hpsa),

Model 1: Geometrical Descriptors Model (2D)

$$
\begin{aligned}
\mathrm{pIC}_{50}= & 1.69-0.01(h p s a)+3.46(f 2 d v s a b)-4.77(f 2 d v s a b u) \\
& -0.014(b b d s a)+0.83(f 2 d v s a b a) \\
& r^{2}=0.80(n=23), q^{2}=0.75(n=20)
\end{aligned}
$$

fraction of two-dimensional van der Waals surface area (2D - VSA) of hydrophobic groups $(f 2 d v s a b)$, fraction of $2 \mathrm{D}$ - VSA of hydrophobic unsaturated groups $(f 2 d v s a b u), \mathrm{H}$ bond donor surface area ( $b b d s a)$ and fraction of 2D-VSA of H-bond acceptor groups (f2dvsaha).

It can be seen that a decrease in the molar hydrophobic surface area would enhance PC3 activity. Although an overall lower molar hydrophobicity is favored, the positive coefficient on f2dvsab means a few hydrophobic groups are still acceptable. A negative coefficient on $f 2 d v s a h u$ shows that a decrease in hydrophobic unsaturated groups' surface area contributes in improving the potency of the compounds. The model also indicates that a decrease in $\mathrm{H}$-bond donors surface area (bbdsa) coupled with complementary increase in surface area of hydrogen bond acceptor groups $(f 2 d v s a h a)$ are greatly favored. Collectively, the results point to a compound that is electronically less bulky or less polarizable, yet big enough to contain hydrophobic groups and hydrogen bond acceptor groups such as rings and electronegative atoms. 
The equation was then validated using the leave-one-out cross-validation method. The initial results involving the 23 compounds gave only a rather less satisfactory correlation between experimental and calculated activity. This is not uncommon, and this has been partly attributed to the quality of the experimental data, which serves as the dependent variable. However, the compounds whose experimental activity was probably taken with poor accuracy can be discriminated using the jackknife method. In jackknife method, the compounds in the dataset $(n$ compounds) are removed one at a time and then $r^{2}$ is obtained for $n-1$ dataset. The compound that gives the best improvement in the $r^{2}$ value is omitted in that cycle. This process can be repeated until no remarkable improvement in the $r^{2}$ value is observed.

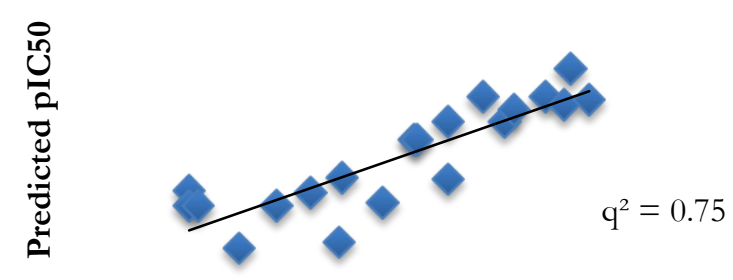

Figure 2. Predicted $p I C_{50}$ values using Geometrical Descriptors Model versus Experimental pIC $C_{50}$ values for 20 curcumin analogues against PC-3 cell line. (Analogues 12, 20 and 21 were eliminated after the jackenife procedure.)

When applied to the dataset of geometrical descriptors, the jackknife procedure yielded a $q^{2}$ of 0.75 with only three compounds $(\mathbf{1 2}, \mathbf{2 0}$, and 21) discriminated. The calculated $\mathrm{pIC}_{50}$ values using the model on geometrical predictors are summarized in Table 2. Gratifyingly, the cross-validation on 20 samples gave a very satisfactory results, considering that the minimum acceptable predictive correlation coefficient is only 0.60 (Figure 2) (Wold, 1991). The scatter plot of the residuals (not shown) also reveals that there is no bias in the error of prediction, the errors being distributed uniformly approximately around zero (i.e. average residual $=-0.02)$.

The model based on geometrical descriptors can be further understood by examining the other models based on some familiar molecular properties. For example, the model constructed from physical descriptors (Equation 2) corroborates the QSAR involving geometrical predictors (Equation 1). Equation 2 clearly unveiled the common physical properties of the compounds that dictate the observed biological activity. The importance of the properties such as polarizability, approximate surface area ( $S A A p$ prox), and volume has been implicitly bared in Equation 1 (vide supra). Moreover, the other two variables $(\log P$ and refractivity) in Equation 2 are also size-dependent.

Table 2. Predicted $\mathrm{pIC}_{50}$ values using Geometrical Descriptors Model, Experimental pIC $_{50}$ values, and Residuals for curcumin analogues against PC-3 cell line.

\begin{tabular}{cccc}
\hline $\begin{array}{c}\text { Curcumin } \\
\text { Analogue }\end{array}$ & $\begin{array}{c}\text { Experimental } \\
\text { pIC }^{50}\end{array}$ & $\begin{array}{c}\text { Predicted } \\
\text { pIC }^{50}\end{array}$ & Residual \\
\hline 1 & -1.60 & -1.11 & 0.49 \\
\hline 2 & -1.44 & -1.46 & -0.02 \\
\hline 3 & -1.60 & -1.20 & 0.40 \\
\hline 4 & -1.57 & -1.20 & 0.37 \\
\hline 5 & -0.88 & -0.80 & 0.08 \\
\hline 6 & -0.77 & -1.04 & -0.27 \\
\hline 7 & -1.11 & -1.03 & 0.08 \\
\hline 8 & -1.12 & -1.42 & -0.30 \\
\hline 9 & -0.87 & -0.80 & 0.07 \\
\hline 10 & -1.32 & -1.20 & 0.12 \\
\hline 13 & -1.21 & -1.12 & 0.09 \\
\hline 14 & -0.59 & -0.69 & -0.10 \\
\hline 15 & -0.77 & -0.69 & 0.08 \\
\hline 16 & -0.98 & -1.18 & -0.20 \\
\hline 17 & -0.46 & -0.54 & -0.08 \\
\hline 18 & -0.40 & -0.59 & -0.19 \\
\hline 19 & -0.56 & -0.62 & -0.06 \\
\hline 22 & -0.38 & -0.37 & 0.01 \\
\hline 23 & -0.32 & -0.56 & -0.24 \\
\hline 24 & -0.66 & -0.54 & 0.12 \\
\hline
\end{tabular}


Model 2: Physical Descriptors Model

$$
\begin{aligned}
\mathrm{pIC}_{50}= & -10.55-0.02(\text { SAApprox })+0.04(\text { Volume })+1.54(\log P) \\
& -0.21(\text { Refractivity })-0.20(\text { Polarizability }) \\
& r^{2}=0.83(n=23), q^{2}=0.63(n=20)
\end{aligned}
$$

The tendency of an electron cloud to be distorted from its normal shape is referred to as its polarizability. The polarizability of an atom (or molecule) depends largely on how diffuse or spread out its electron cloud is. Molar refractivity is a measure of the total polarizability of a mole of a substance and is dependent on the temperature, the index of refraction, and the pressure. Equation 2 tells that a more compact electron distribution would improve the anti-proliferative activity of the curcumin analogue.

$\log P$ is used to describe the lipophilicity of a compound. It is defined as the log of the octanol-water partition coefficient taken from the ratio of the respective concentrations of a compound in the octanol and water partitions of a two-phase system at equilibrium (Leo et al., 1971). Based on our model, it can be deduced that the more lipophilic the compound (higher $\log \mathrm{P}$ ), the more active the curcumin derivative against PC-3. The result of cross-validation of model 2 is displayed in Figure 3. We are pleased to find that the predictive squared correlation coefficient $(0.63)$ is also over the acceptable value for a meaningful QSAR model.

Model 3: Constitutional Descriptors Model (OD)
The other models of the inhibitory activity of curcumin analogues against PC-3 also nicely support the preceding models. The zerodimensional constitutional descriptors model (Equation 3) also features the polarizability parameter common in the two models discussed above.

The negative coefficient of Mean Atomic Polarizability $(M p)$ in Equation 3 is in agreement (i.e. highly correlated) with that of

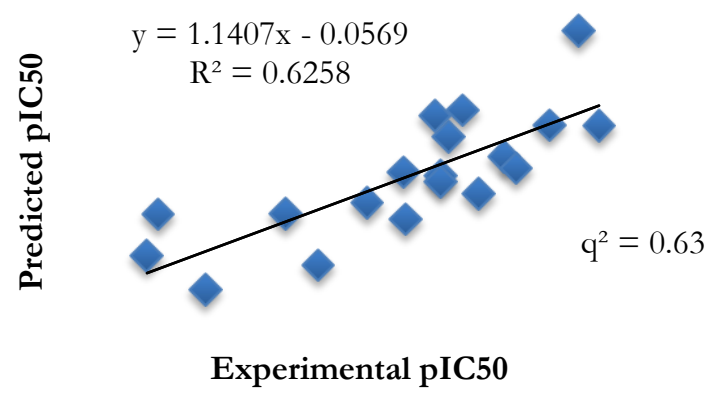

Figure 3. Predicted pIC50 values using Physical Descriptors Model versus Experimental $\mathrm{pIC}_{50}$ values for curcumin analogues against PC-3 cell line. (Analogues 1, 10, 18 were discriminated in the jackknife procedure.)

$$
\begin{aligned}
\mathrm{pIC}_{50}= & 5.181-20.051(M p)-0.182(n B M)+12.724(A R R) \\
& +28.901(\mathrm{RBF})+0.601(n S) \\
& r^{2}=0.75\left(n=23, q^{2}=0.64(n=22)\right.
\end{aligned}
$$

the polarizability parameters in Equations 1 and 2. Decreasing the number of multiple bonds in the compound and increasing rotatable bond fraction $(R B F)$ or saturated bonds have the same effect of reducing the electron density, which parallels polarizability. Increased aromatic ratio (ARR) and number of sulfur atoms $(n S)$ are consistent with larger hydrogen bond acceptor surface area suggested in Model 1. This does not contradict the model on functional group counts (Equation 4), which suggests that the number of hydroxyls (nroh and naroh) be reduced in favor of a bigger $\mathrm{S}$ acceptor group. Likewise, the results of cross-validation of model 3 involving 22 compounds (excluding 7) were satisfactory, the predictive squared correlation coefficient was above the allowable value and the average residual was essentially zero. 
Model 4: Functional Group Counts Model (1D)

$$
\begin{aligned}
\mathrm{pIC}_{50}= & -0.50-0.61(\text { nroh })-0.18(\text { naroh }) \\
& r^{2}=0.708(n=23), \quad q^{2}=0.62(n=23)
\end{aligned}
$$

(Equation 4)

The path/walk descriptor (PW5) in Equation 5 is a shape index. Molecular Path/Walk Index is defined as the average sum of atomic path/walk indices of equal length (Leo et al., 1971). The lopping centric index is defined as the mean information content derived from the pruning partition of acyclic graphs (Todeschini and Consonni, 2000). Both PW5 and Lop are highly correlated with molecular volume, which is an important variable in Equation 2. An increase in TNO (sum of topological distances between $\mathrm{N} \& \mathrm{O}$ ) also implies greater volume and improves potency. Moreover, the hyperdistance path index is highly correlated with refractivity and polarizability, which are ubiquitous in the models.

Model 5: Topological Descriptors Model (2D)

$$
\begin{aligned}
\mathrm{pIC}_{50}= & -3.795+21.703(\mathrm{PW} 5)+1.662(\mathrm{Lop}) \\
& +0.003(\mathrm{TNO})-6.76 \times 10^{-5}(\mathrm{hydp}) \\
& r^{2}=0.75(n=23), \quad q^{2}=0.66(n=21, \text { without } \mathbf{1} \text { and 20) }
\end{aligned}
$$

The electrotopological state (E-state) indices encode both electronic and topological properties of the molecule (Kier and Hall, 1999). The E-state value is a measure of the electron richness of an atom or group of atoms in a molecule. Thus, according to Equation 6, the electron cloud around $\mathrm{CH}_{3}$ $(s s \mathrm{CH} 3)$ and $\mathrm{NH}($ saa NH) groups must be made more available in order to improve the activity. On the other hand, the availability of electrons in oxygen containing groups $(s s \mathrm{OH})$ and $s s O$ ) must be reduced in accord with the functional group count model (Equation 4). To our delight, the negative coefficient of sHydrpus, a group type hydrogen E-state index, which encodes the sum of overall hydrophobic unsaturated groups, exactly represents the $f 2 d v s a b u$ of model 1 .

Model 6: E-state Model (2D)

$$
\begin{aligned}
\mathrm{pIC}_{50}= & 3.213+0.260(s s C H 3)+0.256(\text { saaNH })-0.051(s s \mathrm{OH}) \\
& -0.119(s s O)-0.154(s H y d r p u s) \\
& r^{2}=0.7778(n=23), \quad q^{2}=0.62(n=23)
\end{aligned}
$$

The different models derived here are independent of each other and their derivation was not dependent on other existing models. Each model derived from a class of predictors has its own peculiar strengths and weaknesses. Nevertheless, juxtaposition of all the models displays closely spaced best-fit lines (Figure 4) demonstrating a highly comparable quality of predictions. Moreover, the overall average residual was practically nil $(-0.002)$ and the errors were randomly scattered around zero indicating absence of any bias in the prediction.
Furthermore, it was very pleasing to find that all six models provided common features that curcumin derivatives must possess in order to enhance their potency against PC-3 cell line. Accordingly, new generation of curcumin analogues, as potential antiprostate cancer agents, must have smaller surface area and be less polarizable without sacrificing the volume. These structural requirements may be accomplished by decreasing the number of diffused orbitals from unsaturated bonds and heteroatoms like oxygen. 


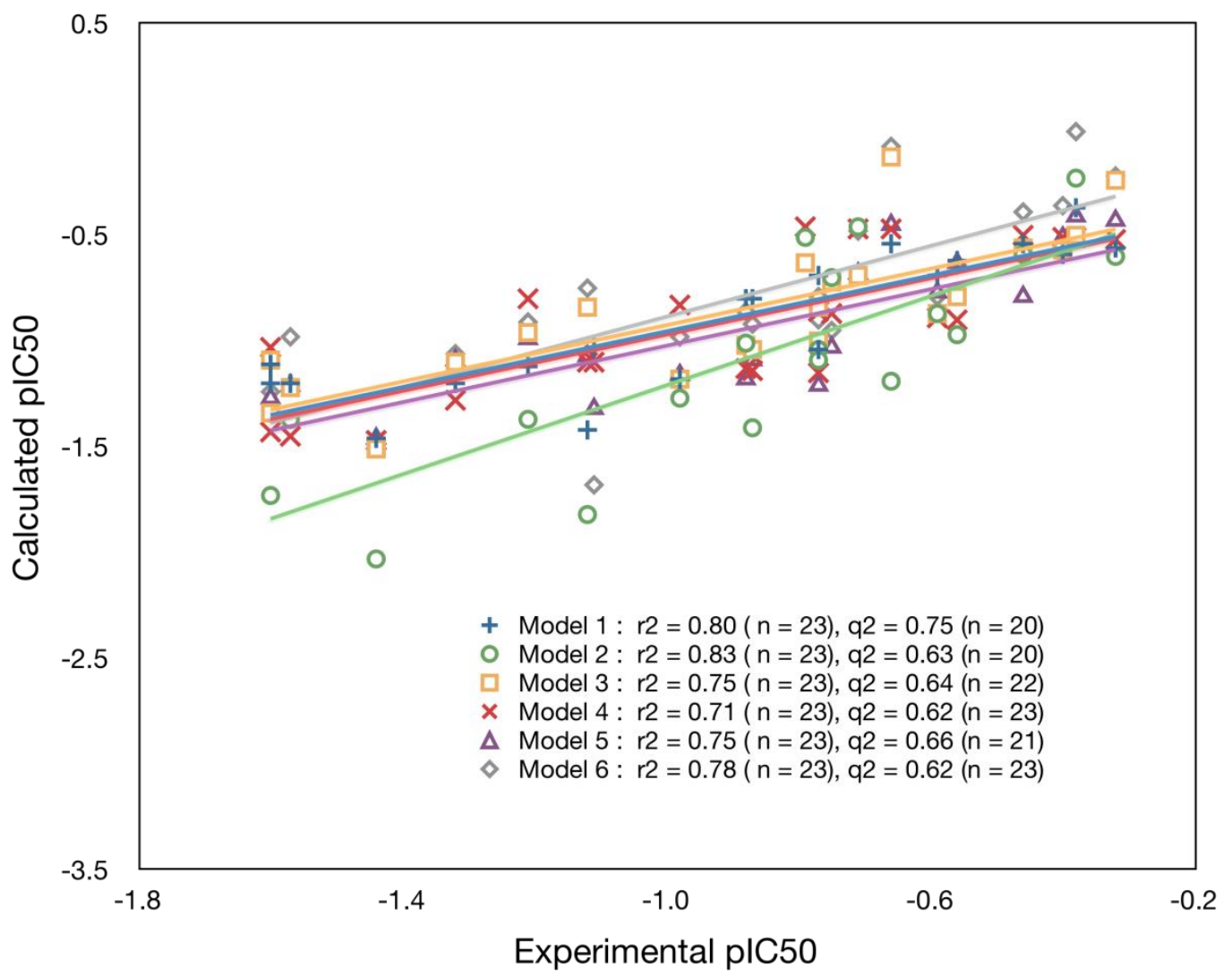

Figure 4. Predicted pIC50 values using the different models versus experimental pIC $C_{50}$ values for curcumin analogues against $P C-3$ cell line.

Each model developed in this work represents a set of molecular properties that are linearly predictive of the negative logarithm of inhibitory activity $\left(\mathrm{pIC}_{50}\right)$ of curcumin derivatives against PC-3 cancer cell lines. The statistically sound models reveal the relevant molecular properties that contribute to the observed biological activity of the compounds. A lower $\mathrm{IC}_{50}$ value is desired because it means lower concentration of inhibitor is needed to effect 50 percent inhibition of cancer. Structural modifications guided by these models would result in an inexpensive and fast design of new generation of curcumin analogues for anticancer chemotherapy. This work is highly valuable in accessing curcumin variants that have lower $\mathrm{IC}_{50}$ values than that of presently known analogues.

As a preliminary attempt to demonstrate the collective observation above, we modified compound $\mathbf{2 3}$, the most active analogue in the dataset. Simple single replacement of a methoxy $\left(\mathrm{CH}_{3} \mathrm{O}-\right)$ group on each aromatic ring with methyl $\left(\mathrm{CH}_{3}\right)$ to yield modified analogue, MA 1, (Figure 5) remarkably improved the calculated $\mathrm{IC}_{50}$ value from 3.52 to $2.87 \mathrm{mM}$ using Equation 1. The removal of two $\mathrm{O}$ atoms certainly reduces the polarizability of the molecule due to the loss of four nonbonding electron pairs, which have otherwise taken up a significant space. Further substitution of methoxy by methyl groups yielded much better $\mathrm{IC}_{50}$ values, albeit the $\log P$ values violate Lipinski rule (Lipinski, 2004) of drugability. On the other hand, we noted that as the number of more polarizable<smiles>COc1cc(C)c(/C=C/C(=O)/C=C/c2c(OC)cc(OC)cc2OC)c(OC)c1</smiles>

Figure 5. Curcumin analogue $\boldsymbol{M A}$ 1, a potentially potent PC-3 inbibitor 
$-\mathrm{OCH}_{3}$ groups was increased the calculated activity corres-pondingly decreased. Inspired by these promising results, more systematic and exhaustive structure modification studies on these compounds are underway in our group.

\section{CONCLUSIONS}

The quantitative structure-activity relationship study on the curcumin analogues against prostate cancer cell lines unveils the specific molecular features that curcumin variants must possess for optimum inhibitory activity. The models encompassing physical, geometrical, constitutional, functional group count, topological, and electrotopological parameters point to a curcumin-based structure with lower surface area, greater molecular volume, low polarizability, greater aromaticity yet lesser multiple bonds. The tight agreement of the different models to one another also demonstrates their validity and fortifies their significance and utility in unraveling the target compound for prostate cancer chemotherapy.

Consequently, complementary experimental structure-activity relationship (SAR) studies guided by the insights from this work are very instructive and are worth pursuing. However, further elaborate modeling studies including support vector machines (SVM) and artificial neural network (ANN) are highly recommended in exploring possible nonlinear relationships involving those discriminated in the jackknife procedure.

\section{REFERENCES}

Aggarwal BB, Kumar A, Bharti AC. Anticancer potential of curcumin: preclinical and clinical studies. Anticancer Res. 2003; 23:363-398.

Anand P, Thomas SG, Kunnumakkara AB, Sundaram C, Harikumar KB, Sung B, et al. Biological activities of curcumin and its analogues (Congeners) made by man and Mother Nature. Biochem Pharmacol. 2008; 76(11):1590-1611.
Campbell FC, Collett GP. Chemopreventive properties of curcumin. Future Oncol. 2005; 1(3):405-414.

Chathoth S, Thayyullathil F, Galadari S. Curcumin Cell Signalling: A Possible Target for Chemotheraphy. Curr Trends Biotechnol Pharm. 2008; 2(2):226-238.

Fuchs JR, Pandit B, Bhasin D, Etter JP, Regan $\mathrm{N}$, Abdelhamid D, et al. Structure-activity relationship studies of curcumin analogues. Bioorg Medicinal Chem Lett. 2009; 19(7):2065-2069.

Gong G. Cross-validation, the jackknife, and the bootstrap: excess error estimation in forward logistic regression. J Am Stat Assoc. 1986; 81(393)108-113.

Hergenhahn M, Soto U, Weninger A, Polack A, Hsu CH, Cheng AL, et al. The chemopreventive compound curcumin is an efficient inhibitor of Epstein-Barr virus BZLF1 transcription in Raji DR-LUC cells. Mol Carcinogen. 2002; 33(3):137-145.

Hypercube, Inc. HyperChem. Version 8.0. Gainesville, FL: Hypercube, Inc.; 2011.

Kier LB, Hall LH. Molecular Structure Description: The Electrotopological State. Lemont B, editor. New York: Academic Press; 1999.

Lee SK, Chang GS, Lee IH, Chung JE, Sung KY, No KT. PreADME: PC-based Program for Batch Prediction of ADME Properties. $15^{\text {th }}$ European Symposium on Quantitative Structure - Activity Relationships \& Molecular Modeling; 2004 Sep 5-11; Istanbul Turkey. Available from: http://preadmet.bmdrc.org/

Leo A, Hansch C, Elkins D. Partition coefficients and their uses. Chem Rev. 1971; 71(6):525-616.

Lipinski CA. Lead- and drug-like compounds: the rule-of-five revolution. Drug Discov Today Technol. 2004; 1(4)337-341. 
Sa G, Das T, Banerjee S, Chakraborty J. Curcumin: From Exotic Spice to Modern Anticancer Drug. Al Ameen J Med Sci. 2010; 3:21-37.

Srivastava RM, Singh S, Dubey SK, Misra K, Khar A. Immunomodulatory and therapeutic activity of curcumin. Int Immunopharmacol. 2011; 11(3):331-341.

Tabachnick BG, Fidell LS. Using Multivariate Statistics. 5th ed. London: Pearson/Allyn \& Bacon; 2006.

Tetko IV, Gasteiger J, Todeschini R, Mauri A, Livingstone D, Ertl $P$, et al. Virtual computational chemistry laboratory-design and description. J Comput Aid Mol Des. 2005; 19(6):453-63.
Thanikaivelan P, Subramanian V, Raghava Rao J, Nair BU. Application of quantum chemical descriptor in quantitative structure activity and structure property relationship. Chem Phys Lett. 2000; 323:59-70.

Todeschini R, Consonni V. Handbook of Molecular Descriptors. Weihheim, Germany: Wiley-VCH Verlag GmbH; 2000.

Wold S. Validation of QSAR's. Quant Struct Act Relat. 1991; 10(3):191-193. 